\title{
Registering Liver Pathological Images with Prior In Vivo CT/MRI Data
}

\author{
Huadong Wu, Alyssa M. Krasinskas, Mitchell E. Tublin, and Brian E. Chapman
}

\begin{abstract}
Liver transplantation affords a unique opportunity to assess and improve radiological imaging of the liver, as the full explanted liver is available for review and comparison. Quantitative comparison between the explanted liver and in vivo images acquired prior to transplantation requires accurate registration of images of the explanted liver to the radiological images. However, this registration problem is challenging because the orientation change and the deformation magnitude between the two image sets exceed the level assumed for most registration algorithms. This paper suggests a two-step registration process to overcome the difficulty: to first align the orientation of 3D liver models built from two sets of image data using maximum volume overlap as their similarity measurement, and second to deform one model to match the other. The key contribution of this paper is that it utilizes the global volumetric information and the asymmetry property of the liver model to determinately provide a simple and reliable initial point for further deformable model based registration. Our experimental data demonstrate the effectiveness of this approach.
\end{abstract}

\section{Introduction}

\subsection{Motivation}

Imaging of the liver is an important problem in radiology, particularly regarding cancer. Not only is the liver an important target for metastatic disease, but the incidence of primary liver tumors, such as hepatocellular carcinoma (HCC), has reach epidemic levels in some parts of the world. The health impact of HCC world-wide is tremendous [8]. However, the early clinical and radiological manifestations of HCCs are subtle and optimizing imaging strategies for detecting them is difficult [9]. We believe that explanted livers provide a unique basis for optimizing MR or CT strategies for imaging the liver, particularly regarding the early detection of HCC.

Correlating in vivo liver images with photographs of the sectioned explanted liver is difficult since the liver geometry changes dramatically when the flexible liver is no longer supported by its surrounding anatomic structures. Additional deformations occur due to loss of tissue distension from blood pressure and tissue relaxation when the liver capsule is cut. However, the liver boundary shape (3D surface) is a relatively rich feature for matching the pathology images to the in vivo images. This paper suggests the overall geometric shape retains sufficient information to do a preliminary alignment of the ex vivo and in vivo images. Application of a deformable registration can then be applied to provide final alignment of the images. 


\subsection{Registration Challenge}

Modeling deformable anatomical structures with significant variability is currently being investigated. Deformable geometric models allow structures to change shape (from static point of view) or to evolve (from dynamic point of view) subject to constraints derived from physical principles. These models provide a reasonable basis for image registration [2], [4]. The most widely used physical assumption for static models is an elastic body, whose substrate responding naturally to applied forces and internal structural constraints. Dynamic models usually assume the viscous fluid property that the applied forces are proportional to the time rate of change in displacement [1], [3].

Almost all registration methods today assume that the initial estimation of location and pose is close to the ground truth so that the optimal point can be assumed to be within a local optimum. Obviously our pathological liver registration situation violates this basic assumption. Therefore, for our liver registration problem we need to align the images so as to find an acceptable initial start in registration.

The generally used approach to initialize the image registration process is to take course-to-fine multiple resolution registration scheme, using the result from the course-resolution registration as the initial point for the finer resolution registration. Ourselin et al. extended this approach (named "Block Matching" method) for rigid body registration, in which they use the majority of sub sets with maximized similarity to estimate a start point [5]. Lau et al. also used a multiscale approach and addressed the initial estimation problem [6]. They build a deformation vector field by matching block sub-regions between the images using a voxel-similarity measure. A median filter is then used to ensure a locally smooth transformation [11].

It is not difficult to conclude that these approaches are not suitable for our liver registration tasks, where orientation is totally lost, large deformation is assumed, and the voxel information from pathological images can be at best sparsely available due to the thick sectioning of the explanted liver ${ }^{1}$.

Intuitively, the best solution to this challenge would require globally abstracting the geometry characteristics of the entity of concern. Using the medial axis description of geometric body, a research topic getting more popular recently, might be a good candidate to realize such abstraction [2]. However, the medial axis extraction process is intrinsically sensitive to surface variation of the object [7], and in our case the constructed 3D models from pathological images are not sufficiently stable in the sense that the slices are arbitrarily laid out ${ }^{2}$ and contour line transitions between adjacent images are not smooth.

This paper will show that, because of the asymmetry characteristics of the liver geometric shape, the geometric principle axis description can be used as a simple but effective clue for the orientation alignment to be used as the initial step in the registration process. Following the gross alignment of the respective volumes using the geometric principle axes, local deformations can easily be applied.

${ }^{1}$ Even if the liver is well cut into slices during the pathology examination, the slices are usually 10 20 mm thick. Therefore, if a 3D volume model is built out of the slices, no real voxel information between slices is available, thus limiting the usefulness of truly $3 \mathrm{D}$ to $3 \mathrm{D}$ deformable registration techniques which are currently available (e.g. www.itk.org).

2 The slices are laid out for photographing in a relaxed shape that resembles their in vivo nature status — but only according to the pathology technician's own imagination. 


\section{Image Registration Via Volume Match}

\subsection{Registration Process Overview}

First, a 3D model is built by segmenting the liver from the CT/MRI data on a slice by slice manner; this model serves as the reference model. The model is interpolated in the slice direction in order to obtain isotropic voxels.

Second, a 3D model is built by stacking the photographs of the sectioned explanted liver, where adjacent slices are aligned via translation, rotation and scaling. Alignment is driven by voxel similarity. This 3D model is subsequently scaled to match the volume of the 3D model built from CT/MRI data.

For these two 3D models, the centroid and voxel distribution are calculated respectively. The voxel distribution is described for each volume using a covariance matrix. Decomposing the two covariance matrices respectively, we can find the geometric principle component axes (eigenvalues) of each object.

Then, by rotating the CT/MRI 3D model around its centroid, we can align the geometric principle axes with respect to the corresponding geometric principle axes of the 3D model built from pathological images.

This global orientation alignment provides an initial point for further model deforming. Currently we correlate pathological slice images with the CT/MRI 3D model's corresponding intersections and do 2D image warping to increase the registration accuracy.

\subsection{Building 3D Models from CT/MRI Images}

The liver CT images are first hand-segmented from the reminder of the images in each cross section slice image, as shown in the left image of Fig. 1. The extracted liver model is represented as a 3-dimensional matrix where the value of pixels outside the liver is set to zero. To describe the overall geometric properties of the liver model $(\Omega)$, we set the liver voxel $V_{i}$ to a unit value and calculate the centroid point coordinates (the mass center as if the liver has unit density)

$$
\left[m_{x}=\frac{\sum_{i \subset \Omega} x_{i} V_{i}}{N}, m_{y}=\frac{\sum_{i \subset \Omega} y_{i} V_{i}}{N}, m_{z}=\frac{\sum_{i \subset \Omega} z_{i} V_{i}}{N}\right], V_{i}=1
$$

and the voxel distribution covariance matrix:

$$
\operatorname{Cov}=\frac{1}{\sum_{i \subset \Omega} V_{i}}\left[\begin{array}{ccc}
\sum_{i \subset \Omega}\left(x_{i}-m_{x}\right)^{2} & \sum_{i \subset \Omega}\left(x_{i}-m_{x}\right)\left(y_{i}-m_{y}\right) & \sum_{i \subset \Omega}\left(x_{i}-m_{x}\right)\left(z_{i}-m_{z}\right) \\
\sum_{i \subset \Omega}\left(y_{i}-m_{y}\right)\left(x_{i}-m_{x}\right) & \sum_{i \subset \Omega}\left(y_{i}-m_{y}\right)^{2} & \sum_{i \subset \Omega}\left(y_{i}-m_{y}\right)\left(z_{i}-m_{z}\right) \\
\sum_{i \subset \Omega}\left(z_{i}-m_{z}\right)\left(x_{i}-m_{x}\right) & \sum_{i \subset \Omega}\left(z_{i}-m_{z}\right)\left(y_{i}-m_{y}\right) & \sum_{i \subset \Omega}\left(z_{i}-m_{z}\right)^{2}
\end{array}\right]
$$

Since the covariance matrix is real, symmetric, and positive semi-definite, it can be eigen-decomposed into principle axes:

$$
\operatorname{Cov}=\mathbf{E} \mathbf{D E} \mathbf{E}^{T}, \quad \mathbf{E}=\left[E_{1}, E_{2}, E_{3}\right]
$$



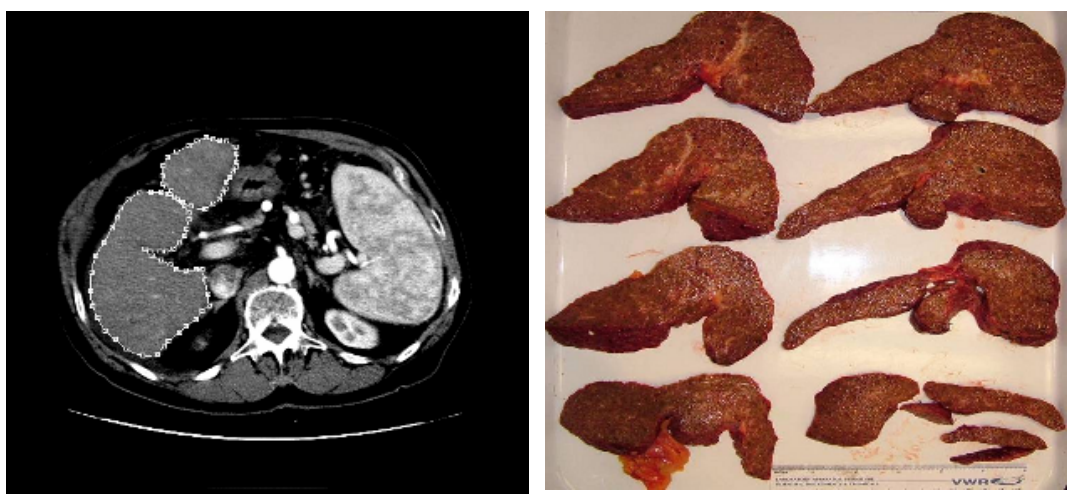

Fig. 1. Left: CT image data with the liver manually segmented; Right: pathological sliced liver images

The eigen vectors, $E_{1}, E_{2}$ and $E_{3}$, describe the 3 orthogonal directions, i.e., the orientation of the geometric principle axes, in which the liver volume spans. The eigen values, or the element $e_{1}, e_{2}$ and $e_{3}$ of the diagonal matrix $\mathbf{D}$, correspondingly describe the voxel distribution in these principle axis directions: the larger the value the more the liver is spread-out in this direction.

\subsection{Building 3D Models from Pathological Liver Slice Images}

We create a 3D model by virtually stacking slices to form a $3 \mathrm{D}$ liver model. The physically analogy of this process is shown in Fig. 2. The images were first preprocessed to segment the liver region, with the background pixel values being set to zero

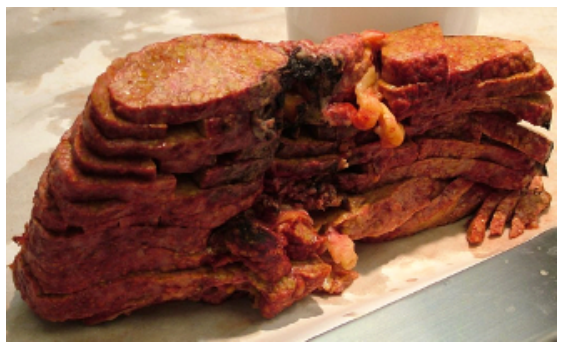

Fig. 2. Pathologists would stack slices in trying to register pathological findings and the voxel dimensions scaled to match the in vivo voxel size. Choosing a slice image that is close to the center of the liver as base, the stack grows out in both directions perpendicular to the base slice image, with local registration between adjacent slice images. Slice-to-slice alignment is achieved via translation, rotation around the center of the ROI (Region of Interest, i.e., the liver pixels inside the image), and isotropic scaling transformation $\mathrm{T}(\cdot)$. This is represented mathematically as:

$$
\left[\begin{array}{l}
x^{\prime} \\
y^{\prime}
\end{array}\right]=\left[\begin{array}{ll}
\lambda & 0 \\
0 & \lambda
\end{array}\right] \cdot\left[\begin{array}{cc}
\cos \theta & -\sin \theta \\
\sin \theta & \cos \theta
\end{array}\right] \cdot\left[\begin{array}{l}
x-m_{x} \\
y-m_{y}
\end{array}\right]+\left[\begin{array}{l}
T_{x}+m_{x} \\
T_{y}+m_{y}
\end{array}\right]
$$

The pixel-wise mean square difference between the image $\mathbf{A}$ in the base stack and the transformed image (the image to be added to the stack) $T(\mathbf{B})$ is used as the metric to optimize the transformation parameters. 


$$
M S(\mathbf{A}, T(\mathbf{B}))=\frac{1}{N} \sum_{i}^{N}\left(A_{i}-B_{i}\right)^{2}
$$

where $A_{i}$ and $B_{i}$ are pixel values in image $\mathbf{A}$ and $\mathbf{B}$, and $N$ is the number of total pixels considered.

To achieve global optimization, the process is implemented in two steps. In the first step, the image pixel values are reduced to binary data with pixels inside or on the liver ROI set to 1 and the background set to 0 . Starting at different initial rotation angles with increment of $10^{\circ}$, a coarse optimization is conducted. Then the best resulting transformation parameters in the first step are used as the starting position in the second step, where a finer optimization step-length is used and the original grayscale image pixel value is used.

With the calculated transformation parameters and by setting the scaling factor back to $\lambda=1$, new slice images are added to the liver model stack. One image at a time, and taking the slice thickness into account, the 3D liver model is built from the 2D pathological images. The volume of the resulting 3D liver model typically differs from the model built from the in vivo CT or MRI images. Since the precise nature of the volumetric changes is unknown, a uniform scaling scheme is used to approximately undo the effect.

We use the same measurements described in Equation 1, 2, and 3 in previous subsection to present the characteristics of the model geometry. The eigen values from the largest to the least are denoted as $e_{1}, e_{2}$, and $e_{3}$, and their corresponding eigenvectors (geometric principle axes) are denoted as $E_{1}^{\prime}, E_{2}^{\prime}$, and $E_{3}^{\prime}$.

\subsection{Aligning the 3D Models}

Even though the 3D model built from in vivo CT scan images may seem substantially different from the 3D model built from the pathological images of the ex-transplanted liver slices, because of the geometric asymmetry, the two models can still be approximately aligned using their geometry principle axis properties. The alignment proceeds as follows. First we rotate the 3D liver model from CT images along the direction vector:

$$
E_{R}=E_{1} \times E_{1}^{\prime}
$$

with an angle:

$$
\theta=\arccos \left(\frac{E_{1} \cdot E_{1}^{\prime}}{\left\|E_{1}\right\| \cdot\left\|E_{1}^{\prime}\right\|}\right)
$$

This aligns the largest geometric principle axis $E_{1}$ of the $3 \mathrm{D}$ liver model from CT images to the direction of the largest principle axis $E_{1}^{\prime}$ of the $3 \mathrm{D}$ liver model from pathological images. Second, the next-to-last geometric principle axis $E_{2}$ of the model is rotated to a new orientation as $E_{R \theta}\left(E_{2}\right)$. Third, we rotate the 3D liver model from CT images along the direction of $E_{1}^{\prime}$ with an angle:

$$
\beta=\arccos \left(\frac{E_{R \theta}\left(E_{2}\right) \cdot E_{2}^{\prime}}{\left\|E_{R \theta}\left(E_{2}\right)\right\| \cdot\left\|E_{2}^{\prime}\right\|}\right)
$$


This rotation aligns the next largest geometry principle axes of the two models. If the two models have a similar geometry shape, their orientation should have now properly aligned in the sense that their second order voxel distribution moment has a maximum overlap. Fourth, since the difference of eigen values corresponding to second and third geometry principle axes are typically less conspicuous, to make sure that the two models are best aligned in terms of volume overlap, fine search is conducted via changing the rotation angle $\beta$.

\subsection{D Image Warping Deformation}

After application of our principle axes alignment and volume scaling, the images are sufficiently aligned to apply a deformable registration technique. For these initial experiments we have implemented a $2 \mathrm{D}$ slice-by-slice deformation using the IDL function WARP_TRI (. ) [10]. Thus our final registration accuracy largely depends on properly choosing the control points in the image warping process.

\section{Results}

Examples of the resulting models after rigid body alignment is completed are shown in Fig. 3. In this example, the geometric variation eigenvalues of the $3 \mathrm{D}$ model built from the in vivo CT images are: $\{3791.8,1265.4,538.6\}$,

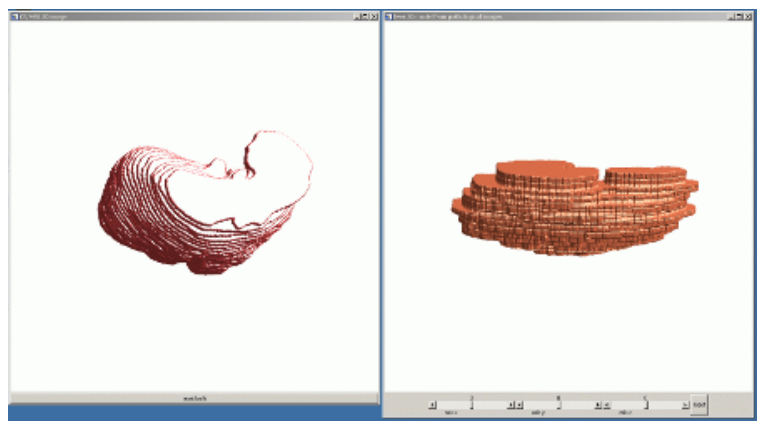

Fig. 3. Two models' orientation aligned and the geometric variation eigenvalues of the volumenormalized 3D model built from the pathological images are $\{5236.0,678.5$, $523.4\}$. It seems that the geometric asymmetry property is largely preserved but the spread-out effect has increased the largest principle vector and decreased the next-largest principle vector.
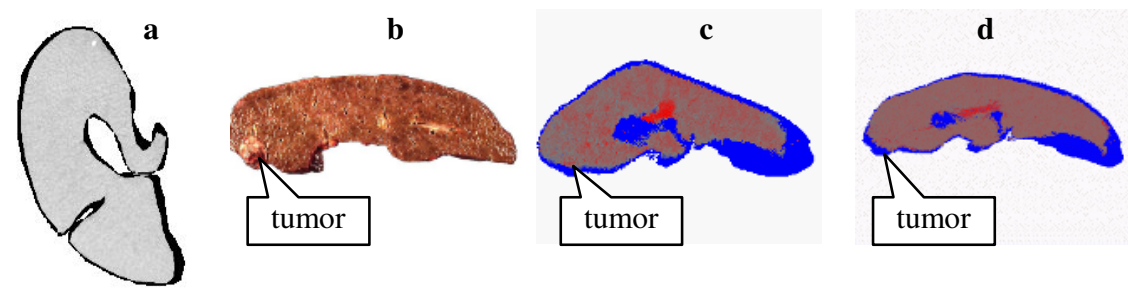

Fig. 4. Pathological liver slice image registration, b: pathological image with a tumor at lowerleft; a: human technician estimated corresponding CT scan slice (a failure); c: global geometry principle axis alignment scheme successfully finds the plane in CT data that contains the tumor; d: registration is improved by simple 2D image warping deformation 
Fig. 4 shows corresponding slices extracted from the rigidly aligned models. It is clear that although the two slices still differ by an in-plane bending, our registration scheme successfully finds the correct region. In this given example, the registration error in the direction parallel to the slice is in the range of $5 \sim 10 \mathrm{~mm}$. Application of the 2D warping provides a nice alignment of the tumor in the two images. We estimated the accuracy of the registration by measuring the displacement between tumor nodules in the in the radiological and pathological images. Using 10 tumors as landmarks, we estimated the residual displacement to be on the order of 10 to $30 \mathrm{~mm}$.

\section{Conclusions and Discussion}

Because of large deformation due to tissue flexibility as well as the lack of consistent fiducial marks between modalities, correlating pathological findings in explanted liver slices to its prior in vivo radiological imaging manifestation poses a big challenge. After a translation to make the centroids coincide, the key to solving this difficult problem is to properly align the geometric orientation of the $3 \mathrm{D}$ model - so that overall correspondence between models can be established and deformable model based registration process can have a proper initial starting point.

The underlying principle for this registration initialization scheme is based on the assumption that although the liver has undergone dramatic deformation after being explanted and sliced for pathological examination, its overall asymmetric shape property is still preserved in terms of the geometric principle axes' orientation. In the cases where this condition fails to be held due to badly laid out slices (i.e., the overall shape is too far away from its natural status) or the slices were not evenly cut, our solution is to manually estimate the approximate orientation and then do a local optimum orientation search based on maximum volume overlap.

When available, a radiological image of the explanted specimen can be extremely useful, because it preserves an intermediate state of deformation (prior to sectioning). Such an ex vivo image can be used as an intermediate target to which the sectioned photographs and in vivo images can be independently matched.

The aligned 3D model pair has global maximum volume overlap. However, the shape deformations that exist between the objects are still large and we believe that a large scale bending needs to be done prior to the application of a typical deformable registration model. We hypothesize that the bending can be estimated by examining the medial axes extracted from smoothed versions of our 3D models. Our results, however, are already good enough for practical visual correlation of the pathological and radiological images.

\section{Acknowledgement}

This work is supported in part by NIH grant CA095759. 


\section{References}

1. D.L.G. Hill, P.G. Batchelor, M. Holden and D.J. Hawkes "Medical Image Registration," Physics in Medicine and Biology vol. 46, no. 3, pp. 1-45, 2001.

2. 2 S.M. Pizer, T. Fletcher, S. Joshi, A. Thall, J.Z. Chen, Y. Fridman and e. al. "Deformable M-Reps for 3D Medical Image Segmentation," International Journal of Computer Vision vol. 55, no. 2/3, pp. 85-106, 2003.

3. T. McInerney and D. Terzopoulos "Deformable Models in Medical Image Analysis: A Survey," Medical Image Analysis vol. 1, no. 2, pp. 91-108, 1996.

4. J. Montagnat, H. Delingette and N. Ayache "A Review of Deformable Surfaces: Topology, Geometry and Deformation," Image and Vision Computing vol. 19, no. 14, pp. 10231040, 2001.

5. S. Ourselin, A. Roche, S. Prima and N. Ayache "Block Matching: a General Framework to Improve Robustness of Rigid Registration of Medical Images," Third International Conference on Medical Image Computing and Computer-Assisted Intervention (MICCAI'00) vol. 1935, pp. 557-566, 2000.

6. Y. Lau, M. Braun and B. Hutton "Non-rigid Image Registration Using a Median-filtered Coarse-to-fine Displacement Field and a Symmetric Correlation Ratio," Physics in Medicine and Biology vol. 46, no. 4, pp. 1297-1319, 2001.

7. S.M. Pizer, K. Siddiqi, J.N. Damon and S. Zucker "Multiscale Medial Loci and Their Properties," International Journal of Computer Vision vol. 55, no. 2-3, pp. 155-179, 2003

8. Hashem B. El-Sera and Andrew C. Mason "Rising Incidence of Hepatocellular Carcinoma in the United States," the New. England Journal of Medecine Volume 340, no. 10, pp. 745-750, March 1999

9. Shahid Hussain, P. Zondervan et al. "Benign versus Malignant Hepatic Nodules: MR Imaging Findings with Pathological Correlation," Radiology Graphics, vol. 22, no. 5, pp. 1023-1039, 2000

10. Robert J. Renka, "Interpolation of Data on the Surface of a Sphere and Triangulation and Interpolation at Arbitrary Distributed Points in the Plane," ACM Transactions on Mathematical Software, Vol.10, no. 4, pp.437-439, Dec. 1984 